Original article

\title{
Intergenerational Continuity in Cannabis Use: The Role of Parent's Early Onset and Lifetime Disorder on Child's Early Onset
}

\author{
Kimberly L. Henry, Ph.D. ${ }^{\mathrm{a}, *}$, and Megan B. Augustyn, Ph.D. ${ }^{\mathrm{b}}$ \\ a Department of Psychology, Colorado State University, Fort Collins, Colorado \\ ${ }^{\mathrm{b}}$ Department of Criminal Justice, College of Public Policy, The University of Texas at San Antonio, San Antonio, Texas
}

Article history: Received March 31, 2016; Accepted September 2, 2016

Keywords: Cannabis abuse and dependence; Cannabis disorder; Early onset; Intergenerational continuity

\section{A B S T R A C T}

Purpose: Children's early onset of cannabis use was examined as a function of their parent's early onset of cannabis and subsequent incidence of a lifetime cannabis abuse or dependence disorder. Methods: Prospective, longitudinal data from the Rochester Youth Development Study and the Rochester Intergenerational Study for 442 parent-child dyads (274 father-child, 168 mother-child) were used. The children were evenly split by sex. Logistic regression models and a path analysis were estimated to assess the effect of parent's cannabis use on child's onset of cannabis by age 15 . Results: Fathers who began using cannabis by age 15 were more likely to meet the criteria for a lifetime cannabis disorder (odds ratio $=5.66,95 \%$ confidence interval $=1.89-16.90$ ). The offspring of fathers who met the criteria for a disorder had higher odds of early initiation of cannabis use (odds ratio $=9.70,95 \%$ confidence interval $=3.00-31.34$ ). Early-onset cannabis use by father was indirectly associated with their child's onset of cannabis use via father's lifetime cannabis disorder. No significant effects for mothers were observed, although analyses were limited due to the low rate of mothers who met the criteria for a lifetime cannabis disorder.

Conclusions: This study provides evidence of intergenerational continuity in cannabis use among fathers and their children and confirms the need to consider timing of use and intervening mechanisms in the study of continuity in cannabis use across generations.

(c) 2016 Published by Elsevier Inc. on behalf of Society for Adolescent Health and Medicine.

\begin{abstract}
IMPLICATIONS AND CONTRIBUTION

Prospective, longitudinal, and intergenerational data were used to examine intergenerational continuity in cannabis use. Intergenerational continuity was observed for fathers. Early-onset cannabis use was indirectly associated a child's early onset of cannabis use via the presence of a lifetime cannabis disorder.
\end{abstract}

Echoing a political climate calling for further legalization of cannabis, the use of cannabis (for nonmedical reasons) is on the rise in the United States [1]. Currently, over $30 \%$ of young adults report recent use of cannabis [2], and $40 \%$ of high school students report a lifetime prevalence of cannabis use [3]. Moreover, nearly $16 \%$ of eighth graders indicate they previously tried cannabis [1]. Despite evidence that cannabis use is becoming increasingly normative, its use by young people can change developmental

\footnotetext{
Conflicts of Interest: The authors have no conflicts of interest to disclose.

* Address correspondence to: Kimberly L. Henry, Ph.D., Department of Psychology, Colorado State University, 220 Behavioral Sciences Building, Fort Collins, CO 80525.

E-mail address: kim.henry@colostate.edu (K.L. Henry).
}

trajectories toward maladaptation [4] with evidence of shortterm (e.g., impulsivity, morbidity, risky sexual behavior, and delinquency) and long-term (e.g., academic underachievement, employment instability, mental illness, psychosocial adjustment, brain development, family functioning) consequences [4-6].

In this study, we focus on early onset of cannabis use, specifically, use that begins by age 15 . The timing of onset of cannabis use may be particularly salient to the harmful consequences of use. For example, early onset of cannabis use is linked to subsequent drug abuse and/or dependence [7-10]. In one study [7], individuals who used cannabis during adolescence were two to four times more likely to display symptoms of cannabis dependence [11] compared with individuals who delayed use until after age 18 . 
Given the salience of early-onset cannabis use, a more thorough understanding of the predictors of the early onset of cannabis is needed. Adopting a life course perspective that stresses the importance of interdependent lives across generations [12], we focus on a generally understudied influence-intergenerational (IG) continuity in cannabis use. This type of continuity is defined by similarity in behavior between parent and child during the same developmental period (e.g., adolescence) [13]. We examine the role of a parent's use of cannabis during his/her own adolescence, and his/her subsequent abuse or dependence, on his/her child's early onset of cannabis use. Surprisingly little published work is available to quantify the specific direct and indirect effects of a parent's cannabis use during adolescence on offspring's cannabis use, particularly when limited to papers meeting the rigor to study IG hypotheses [14]. One exception is Knight et al. [15] who report a direct relationship between frequency of parental use of cannabis during both adolescence and emerging adulthood and frequency of child cannabis use during these same periods. Kerr et al.[16] also examined the relationship between frequency of parent cannabis use during adolescence (retrospectively reported for mothers but prospectively reported for fathers) and offspring cannabis use onset during adolescence and found an indirect IG effect via two social context variables-peer marijuana use and peer delinquency. Most recently, Bailey et al. [17] reported that frequency of parent's current use of cannabis predicted child's use of cannabis, but frequency of parent's cannabis use during late adolescence and early adulthood (historical use) did not. These three studies provide important knowledge on the topic of IG continuity in cannabis use, but more work is clearly needed.

In this study, we add to the scant literature on this topic by using a prospective, longitudinal, and multigenerational data set. We first examine whether the early onset of cannabis use by an adolescent is a function of his/her parent's early onset of cannabis use (i.e., a direct assessment of IG continuity). We then account for a particularly pernicious consequence of early onset cannabis use-a parent's subsequent cannabis abuse or dependence disorder (based on Diagnostic and Statistical Manual, Edition 4 diagnostic criteria [11])-and examine whether the presence of a cannabis disorder serves as an intermediate variable between parental early onset of cannabis use and child onset of cannabis use. Specifically, we test four hypotheses:

1. A parent who began using cannabis by age 15 is more likely to meet the criteria for a lifetime cannabis disorder by adulthood.

2. A child is more likely to initiate cannabis use by age 15 if his/her parent initiated cannabis use by age 15 .

3. A child is more likely to initiate cannabis use by age 15 if his/her parent met the criteria for a lifetime cannabis disorder.

4. The effect of early onset cannabis use by a parent on his/her child's early onset of cannabis use is mediated by the parent's lifetime incidence of a cannabis disorder.

In any study of IG continuity, it is important to acknowledge the sex of the parent because life events of males and females differentially impact the next generation [18], and parental roles tend to vary for mothers and fathers [19]. Evidence suggests that the antisocial behavior of fathers and mothers differentially affect children with greater continuity in antisocial between fathers and their children compared with mothers and their children [18,20-22]. Therefore, we depart from prior work on IG continuity in cannabis use [15-17] and evaluate our hypotheses separately for mothers and fathers to determine if sex of the parent conditions IG continuity in cannabis use.

\section{Methods}

Sample

The data for this study come from two longitudinal, companion studies. The original study, the Rochester Youth Development Study (RYDS), began in 1988 and the IG extension, the Rochester Intergenerational Study (RIGS), began in 1999. Detailed information about the designs of these studies is presented elsewhere; [23] only a brief summary is provided here.

The original RYDS sample of 1,000 adolescents (referred to as $\mathrm{G} 2$; their primary caregiver is referred to as $\mathrm{G} 1$ ) is representative of the seventh- and eighth-grade public school population of Rochester, New York, in 1988. Youth at high risk for antisocial behavior were overrepresented by oversampling males and residence in high-crime areas of the city. RYDS participants completed regular interviews in school or home every six months from 1988 to 1992 (phase 1), annually from 1994 to 1996 (phase 2), and biannually from 2003 to 2006 (phase 3). In general, sample retention was good and analyses reveal attrition did not bias the sample [24].

Beginning in 1999, RIGS selected G2s oldest biological child, referred to as G3, and added new firstborns to the G3 sample in each subsequent year. Both G2 and G3s other primary caregiver completed annual interviews since the inception of RIGS (continuing until G3 turns/turned 18) and G3 completed annual interviews once he/she turned eight. To date, there are prospective, longitudinal data on 529 parent-child dyads. The present analysis uses data from 442 parent-child dyads (274 father-child and 168 mother-child); this includes all dyads in which G3 was born before 2001 (allowing them to have been at least 15 years old at the last available wave of data collection-2016). The children were evenly split by sex. Based on G2's report, when G3 was 15 years old, $96 \%$ of mothers and $67 \%$ of fathers either lived with their child or their child regularly spent the night with them. All data collection procedures were approved by the University at Albany's Institutional Review Board.

\section{Measures}

Early-onset cannabis use by G2 was measured during phase 1 of RYDS and by G3 during RIGS. At the first interview for G2, and during the age 8 interview for G3, respondents reported if they ever used cannabis, and if they had, at what age they started using. In subsequent interviews, respondents reported if they used cannabis since the last interview. Using this information, we created a binary indicator of early onset cannabis use, where the respondent is coded 1 if he/she initiated use of cannabis at or before their age 15 interview and 0 if they initiated use after the age 15 interview (or not at all). We selected cannabis use onset at or before age 15 because this is in line with previous research identifying problematic outcomes as a result of onset at this age [25] and standards set by the Substance Abuse and Mental Services Association for early experimentation and uptake [26].

Lifetime cannabis abuse and dependence was measured when G2 was in their late 20s or early 30s (phase 3) using the Computerized Diagnostic Interview Schedule Version IV (CDIS-IV [27]) during the participant's annual interview. The CDIS-IV is 
Table 1

Descriptive statistics for background covariates

\begin{tabular}{|c|c|c|c|c|c|c|c|c|c|c|c|c|}
\hline \multirow[t]{2}{*}{ Variable } & \multicolumn{3}{|c|}{ Full RYDS sample } & \multicolumn{3}{|c|}{ All known parents } & \multicolumn{3}{|c|}{ Full RIGS sample } & \multicolumn{3}{|c|}{ RIGS subsample } \\
\hline & $\mathrm{N}$ & Mean & SD & $\mathrm{N}$ & Mean & SD & $\mathrm{N}$ & Mean & SD & $\mathrm{N}$ & Mean & SD \\
\hline G2 male & 1,000 & .73 & & 727 & .70 & & 529 & .65 & & 442 & .62 & \\
\hline G2 black & 1,000 & .68 & & 727 & .69 & & 529 & .72 & & 442 & .74 & \\
\hline G2 Hispanic & 1,000 & .17 & & 727 & .17 & & 529 & .15 & & 442 & .16 & \\
\hline G2 age at start of study & 1,000 & 13.57 & .77 & 727 & 13.57 & .76 & 529 & 13.55 & .76 & 442 & 13.61 & .76 \\
\hline Arrest rate (per 100) in G2's neighborhood & 1,000 & 4.19 & 2.07 & 727 & 4.31 & 2.05 & 529 & 4.35 & 2.04 & 442 & 4.46 & 2.03 \\
\hline Proportion of G2's neighborhood in poverty & 1,000 & .33 & .14 & 727 & .34 & .14 & 529 & .34 & .14 & 442 & .35 & .13 \\
\hline G1 years of education & 992 & 11.35 & 2.15 & 724 & 11.26 & 2.16 & 527 & 11.20 & 2.07 & 440 & 11.04 & 2.04 \\
\hline G1 low income status & 901 & .51 & & 678 & .54 & & 499 & .56 & & 415 & .58 & \\
\hline G2 lived with both biological parents & 958 & .24 & & 712 & .22 & & 518 & .24 & & 432 & .21 & \\
\hline G2 age at first birth & 703 & 21.89 & 4.94 & 703 & 21.89 & 4.94 & 529 & 21.72 & 4.71 & 442 & 20.06 & 2.88 \\
\hline G2 early onset & 978 & .24 & & 727 & .26 & & 529 & .27 & & 442 & .29 & \\
\hline G2 cannabis disorder & 678 & .06 & & 574 & .06 & & 490 & .05 & & 411 & .05 & \\
\hline
\end{tabular}

Full RYDS sample refers all G2 participants in the RYDS study; all known parents refers to all G2 participants in the RYDS study who are known to have had a biological child; full RIGS sample refers to all G2s who have taken part in RIGS; RIGS subsample refers to all G2s in the RIGS study who have a biological child (G3) born before 2001. RYDS = Rochester Youth Development Study; RIGS = Rochester Intergenerational Study; SD = standard deviation.

based on the Diagnostic and Statistical Manual, Edition 4, [11] criteria for lifetime substance use, abuse, and dependence. Participants who met the criteria for either lifetime cannabis abuse or dependence (referred to in the results as a disorder) were assigned 1; those who did not meet the criteria for either abuse or dependence were assigned 0 .

We include a set of control variables that are proposed to be causally before G2 early onset of cannabis use and their subsequent cannabis disorder status: G2 sex; G2 race/ethnicity; G2 age at the start of RYDS; G1's years of education; structure of G2's family at the start of RYDS (a binary variable indicating respondents who lived with both biological parents); low income status of G2's family at the start of RYDS (a binary indicator of poverty level income, receipt of welfare, or unemployment of the primary wage earner). We also controlled for two variables describing the neighborhood where G2 lived at the start of RYDS: (1) the arrest rate per 100 people based on Rochester Police Records; (2) the proportion of residents in the census tract living in poverty (from US Census Records). Finally, we control for three variables that were not causally before G2 cannabis use but are important when modeling G3 cannabis use: G3 sex, G2 age at G3's birth, and the average contact of G2 with G3 from age 10 to 15 . The contact variable was created from a two-part question asked of both G2 and G3. First, the respondent was asked if they lived with the other at the time of the interview. If they did not live with the other, then they were asked how frequently they spent time together. For G2, this frequency measure was captured on a five-point scale $(0=$ never, $1=$ once or twice per year, $2=$ less than once per month, 3 = at least once a month, and $4=$ at least once a week). For G3, this frequency measure was captured on a four-point scale $(0=$ never, $1=$ almost never, $2=$ sometimes, and $3=$ often). We modified this frequency measure by assigning those who lived together a score 1 point higher than the maximum(i.e., 5 for the G2 measure and 4 for the G3 measure). We then created a standardized score at each age. The final contact score was formed by averaging the scores across reporters and years.

Descriptive statistics are presented in Table 1 (for demographic variables) and Table 2 (for cannabis variables). Also in Table 1, we present information to ascertain how the sample used for the current analysis differs from the full RYDS and RIGS samples in terms of the demographic variables.

\section{Analysis}

All analyses were conducted in Mplus, version 7.4 [28]. Hypothesis 1 through 3 was assessed using a logistic regression model. Hypothesis 4 was estimated as a path analysis. A weighted least squares means and variances adjusted probit estimator was used to estimate the mediation model [29]. To properly account for missing data, we created 10 multiply imputed data sets in Mplus. All analyses were performed on each of the imputed data sets and the results were pooled using the procedures outlined by Rubin [30].

\section{Results}

\section{Findings for G2 males}

We first considered the relationship between a G2 father's early use of cannabis and the odds that G2 fathers met the

Table 2

Descriptive statistics for cannabis use variables

\begin{tabular}{|c|c|c|c|c|c|c|c|c|c|}
\hline & \multicolumn{3}{|l|}{ G2 } & \multicolumn{3}{|l|}{ G2 } & \multicolumn{3}{|l|}{ G3 } \\
\hline & \multicolumn{3}{|c|}{ Early onset } & \multicolumn{3}{|c|}{ Disorder } & \multicolumn{3}{|c|}{ Early onset } \\
\hline & No & Yes & Missing & No & Yes & Missing & No & Yes & Missing \\
\hline \multicolumn{10}{|l|}{ G2 males } \\
\hline $\mathrm{N}$ & 204 & 70 & 0 & 232 & 17 & 25 & 223 & 35 & 16 \\
\hline Percent among observed cases & $74.5 \%$ & $25.6 \%$ & & $93.2 \%$ & $6.8 \%$ & & $86.4 \%$ & $13.6 \%$ & \\
\hline \multicolumn{10}{|l|}{ G2 females } \\
\hline $\mathrm{N}$ & 112 & 56 & 0 & 159 & 3 & 6 & 138 & 24 & 6 \\
\hline Percent among observed cases & $66.7 \%$ & $33.3 \%$ & & $98.2 \%$ & $1.9 \%$ & & $85.2 \%$ & $14.8 \%$ & \\
\hline
\end{tabular}


Table 3

Cross tabulations of cannabis use variables

\begin{tabular}{|c|c|c|c|c|c|c|c|c|}
\hline \multicolumn{9}{|l|}{ G2 Males } \\
\hline \multirow{2}{*}{$\frac{\text { Panel A }}{\text { G2 early }}$} & \multicolumn{2}{|l|}{ G2 disorder } & \multirow{2}{*}{$\frac{\text { Panel B }}{\text { G2 early }}$} & \multicolumn{2}{|c|}{ G3 early onset } & \multirow{2}{*}{$\frac{\text { Panel C }}{\text { G2 disorder }}$} & \multicolumn{2}{|c|}{ G3 early onset } \\
\hline & No & Yes & & No & Yes & & No & Yes \\
\hline No & 179 (96.8\%) & $6(3.2 \%)$ & No & 169 (89.0\%) & $21(11.1 \%)$ & No & 198 (89.6\%) & $23(10.4 \%)$ \\
\hline Yes & 53 (82.8\%) & $11(17.2 \%)$ & Yes & 54 (79.4\%) & $14(20.6 \%)$ & Yes & $8(50.0 \%)$ & $8(50.0 \%)$ \\
\hline \multicolumn{9}{|c|}{ G2 Females } \\
\hline Panel D & \multicolumn{2}{|l|}{ G2 disorder } & Panel E & \multicolumn{2}{|c|}{ G3 early onset } & Panel F & \multicolumn{2}{|c|}{ G3 early onset } \\
\hline G2 early & No & Yes & G2 early & No & Yes & G2 disorder & No & Yes \\
\hline No & 107 (99.1\%) & $1(.9 \%)$ & No & $91(85.1 \%)$ & $16(15.0 \%)$ & No & $132(84.6 \%)$ & $24(15.4 \%)$ \\
\hline Yes & 52 (96.3\%) & $2(3.7 \%)$ & Yes & 47 (85.5\%) & $8(14.6 \%)$ & Yes & $3(100 \%)$ & $0(0 \%)$ \\
\hline
\end{tabular}

Cells contain the $\mathrm{N}$, with the percentage of the row in parentheses.

criteria for a lifetime cannabis disorder. As illustrated in Table 2, $25.6 \%$ of G2 males used cannabis by age 15 , and $6.8 \%$ met the criteria for a disorder. A higher proportion of those who used cannabis by age 15 met the criteria for a cannabis disorder in adulthood (17.2\%) compared with G2 males who did not use cannabis by age 15 (3.2\%). Supporting hypothesis 1 , the association between male G2 early-onset cannabis use and male G2 lifetime cannabis disorder was significant, adjusting for G2 background variables; the odds of a disorder were over five times higher among men who initiated use of cannabis by age 15 (odds ratio $[\mathrm{OR}]=5.66,95 \%$ confidence interval $[\mathrm{CI}]=1.89-16.90)$.

Regarding IG continuity in early-onset use, early-onset cannabis use was more prevalent among G3 participants whose father began using cannabis by age 15 (20.6\%) compared with G3 participants whose father delayed cannabis use until after age 15 or not at all (11.1\%). However, adjusting for all background variables, a father's early onset cannabis use was not directly associated with G3 early-onset cannabis use (OR $=1.83$, $95 \% \mathrm{CI}=.84-4.03)$. On the other hand, early-onset cannabis use was more prevalent among $\mathrm{G} 3$ participants whose father met the criteria for a lifetime cannabis disorder (50\%) than among G3 participants whose father did not meet criteria for a disorder (10.4\%). A father's cannabis disorder was directly associated with G3 cannabis onset ( $\mathrm{OR}=9.70,95 \% \mathrm{CI}=3.00-31.34)$ after adjusting for all background variables.

Finally, we tested the path analysis to examine the extent to which IG continuity in early-onset use was indirect via a father's lifetime cannabis disorder. The results of our path analysis reveal that male G2 early-onset cannabis use was indirectly associated with G3 early-onset cannabis use via the father's lifetime cannabis disorder (weighted least squares means and variances adjusted probit estimate for indirect path [product of coefficients method $]=.64,95 \% \mathrm{CI}=.15-1.13)$. In this analysis, a 95\% CI that does not contain 0 is statistically significant $(p<.05)$ and is indicative of a significant indirect IG pathway. A father's early onset of cannabis use increased the likelihood that he experienced a cannabis disorder, which subsequently increased the likelihood that G3 initiated use of cannabis at an early age. Therefore, while hypothesis 2 was not supported, hypotheses 1 , 3 , and 4 were supported for G2 males.

\section{Findings for G2 females}

Among G2 females, 33.3\% used cannabis by age 15, and 1.9\% met the criteria for a lifetime cannabis disorder. Given the small number of G2 females who met the criteria for a disorder (three females), only hypothesis 2 was tested. We found no evidence of IG continuity for mothers. Early-onset cannabis use was no more prevalent among G3 participants whose mother began using cannabis by age 15 (14.6\%) compared with G3 participants whose mother delayed cannabis use to after age 15 or not at all (15.0\%). Adjusting for the background covariates, G2 early onset of cannabis was not significantly associated with G3 early onset of cannabis ( $\mathrm{OR}=.77,95 \% \mathrm{CI}=.25-2.33$ ). In sum, hypotheses 1,3 , and 4 were not tested given the very small number of G2 mothers who met the criteria for a cannabis disorder, and we found no evidence to support hypothesis 2 .

\section{Discussion}

Our research adds to the scientific and public health body of knowledge regarding the "human costs" [31] of drug use as it further demonstrates that the early onset of cannabis use by boys is associated with an increased risk of experiencing a cannabis disorder. Furthermore, we demonstrate that the human costs of cannabis use are not limited to one's own developmental trajectory; a father's use of cannabis is associated with his child's eventual early uptake of cannabis use. We find no evidence of IG continuity in early onset use of cannabis for mothers and their children, and we were prevented from considering mother's more serious cannabis use because so few G2 females in the sample met the criteria for a disorder.

Our study is one of few that considers IG continuity in cannabis use, and, in doing so, we make valuable contributions to the existing literature. It is important however to recognize the limitations of our study. First, the sample size used in this research is relatively small ( $\mathrm{N}=442$ ), and, when separated by G2 sex, the analytic samples become even smaller. The small sample sizes also precluded our ability to consider differential effects as a function of G3 sex and presented problems when considering the role of a G2 cannabis disorder in cannabis continuity among G2 mothers. Although our work is informative with respect to IG continuity in cannabis use for fathers, we were unable to fully study IG continuity in cannabis use among mothers. At this point, we refrain from saying that there is no evidence of IG continuity in cannabis onset among mothers; this would be unwise given the initial sampling strategy of RYDS (i.e., only $27 \%$ were female), and the low prevalence of cannabis disorder among this sample of women. However, the lack of a relationship between early onset of cannabis use and a subsequent disorder is interesting in and of itself. It could be a result of antisocial behavior being more serious and exhibiting more stability across the life course 
among males compared to females [32] or that early onset is more important in the etiology of a cannabis disorder for males than females [33]. It is also important to recognize that a mother's use of other substances (e.g., tobacco and alcohol use) plays an important role in the onset of substance use among her children [34], and it may be that more visible substance use (e.g., tobacco or alcohol) is more relevant to the study of continuity in substance use among mother-child dyads as illegal drug use may be hidden from children. Nevertheless, additional research is needed to speak to these possibilities.

Another limitation arises from the IG nature of the study. Currently, G3s born to parents who delayed parenthood into their late 20 s or older are not represented in this research because their firstborns have not yet aged into adolescence (at least not sufficiently so to be included in the analyses presented here). It will be important to revisit these analyses when the full sample of G3s has passed through adolescence.

Finally, it is important to note that the Rochester studies represent families who lived in Rochester, New York, in the mid 1980s, and the extent to which these findings generalize to other samples is unknown. However, we note that almost all other prospective, longitudinal data sources are limited in the same way with respect to a specific geographic locale. Nonetheless, this is the first study to demonstrate IG continuity among a predominantly minority sample (i.e., $90 \%$ of the families were nonwhite in RIGS compared with $22 \%$ in the Oregon Youth Study [16], 27\% in the National Youth Survey Family Study [15], and 59\% in the Seattle Social Development Study Intergenerational Project [17]), suggesting the importance of IG continuity in an ethnically diverse sample.

Notwithstanding the lack of IG continuity in cannabis onset for females, this research has significant implications for policy as it draws attention to the importance of mitigating consequences of early onset of cannabis use to limit human costs (i.e., escalation of use to a disorder and the ill consequences associated with abuse) and protect the next generation. Attempts to prevent or delay the onset of cannabis use are now even more imperative to the future well-being of society given the recent legalization of cannabis in some locales, the likely expansion of legalization across the United States, and the overall increased visibility of cannabis to youth. This research points to a father's use of cannabis, in terms of early onset and a subsequent cannabis disorder, as a factor that puts his child at increased risk for early onset of cannabis use. Therefore, preventing the early onset and escalation of cannabis use and/or mitigating the ill effects of cannabis use will have important implications for the next generation of children.

There are a host of effective universal prevention programs (targeting the general population) aimed at reducing the likelihood of drug use onset, including cannabis, in late childhood and early adolescence such as Positive Action [35] and Life Skills Training [36]. Moreover, effective selective intervention programs like Project Towards No Drug Abuse [37] can target adolescents who experienced an early onset of cannabis use to prevent subsequent problematic use. In addition, efforts to help fathers who suffered or are suffering from a cannabis disorder recognize that their children are at an increased risk for early onset of cannabis use may also prove fruitful, particularly if fathers can be given the tools to help prevent the use of cannabis by their children. Indeed, there are effective interventions designed to assist parents in this way such as The Family Check-Up [38] and The Strengthening Families Program [39].
The study presented here is largely descriptive and sets the stage for important future work. We did not attempt to isolate the specific causal mechanism(s) accounting for the transmission of risk across generations (e.g., heritability, imitation or modeling, transmission of risk factors that promote drug use, etc.), nor did we examine potential moderators of IG continuity other than parental sex. The next step for research is to unpack the mechanisms by which parents' experiences with cannabis influence their children's experiences with cannabis.

\section{Uncited Table}

Table 3.

\section{Acknowledgments}

The authors thank Rebekah Chu, Ph.D., for her assistance in compiling the data set for the analyses presented in this manuscript.

\section{Funding Sources}

Support for the Rochester Youth Development Study has been provided by the Centers for Disease Control and Prevention (R01CE001572), the Office of Juvenile Justice and Delinquency Prevention (2006-JW-BX-0074, 86-JN-CX-0007, 96-MU-FX-0014, 2004-MU-FX-0062), the National Institute on Drug Abuse (R01DA020195, R01DA005512), the National Science Foundation (SBR-9123299), and the National Institute of Mental Health (R01MH56486, R01MH63386). Work on this project was also aided by grants to the Center for Social and Demographic Analysis at the University at Albany from NICHD (P30HD32041) and NSF (SBR-9512290).

\section{References}

[1] National Institute on Drug Abuse. Monitoring the Future 2015 Survey Results. Available at: https://www.drugabuse.gov/related-topics/trendsstatistics/infographics/monitoring-future-2015-survey-results. Published 2015. Accessed March 28, 2016.

[2] Johnston LD, O’Malley PM, Bachman JG, Schulenberg JE. Monitoring the future national results on drug use: 2012 Overview, Key findings on adolescent drug Use. Ann Arbor Inst Soc Res Univ Michigan; 2013.

[3] Johnston LD, Miech RA, O'Malley PM, et al. Use of Ecstasy, Heroin, Synthetic marijuana, alcohol, Cigarettes Declined among US Teens in 2015. Ann Arbor. 2015. Available at: http://www.monitoringthefuture.org.

[4] Washburn IJ, Capaldi DM. Heterogeneity in men's marijuana use in the 20s: Adolescent antecedents and consequences in the 30s. Dev Psychopathol 2014;27:1-13.

[5] Lisdahl KM, Gilbart ER, Wright NE, Shollenbarger S. Dare to delay? The impacts of adolescent alcohol and marijuana use onset on cognition, brain structure, and function. Front Psychiatry 2013;4:53.

[6] Volkow ND, Swanson JM, Evins AE, et al. Effects of cannabis use on human behavior, including cognition, motivation, and psychosis: A review. JAMA Psychiatry 2016;73:292-7.

[7] Chen C, Storr CL, Anthony JC. Addictive behaviors early-onset drug use and risk for drug dependence problems. Addict Behav 2009;34:319-22.

[8] DeWit DJ, Hance J, Offord DR, Ogborne A. The influence of early and frequent use of marijuana on the risk of desistance and of progression to marijuana-related harm. Prev Med 2000;31:455-64.

[9] Ehlers CL, Gizer IR, Vieten C, et al. Cannabis dependence in the San Francisco Family Study: age of onset of use, DSM-IV symptoms, withdrawal, and heritability. Addict Behav 2010;35:102-10.

[10] Windle M, Windle RC. Early onset problem behaviors and alcohol, tobacco, and other substance use disorders in young adulthood. Drug Alcohol Depend 2012;121:152-8.

[11] American Psychiatric Association (APA). Diagnostic and statistical Manual of mental disorders: DSM-IV-TR®; 2000. 
[12] Elder GHJ. Perspectives on the life course. In: Life Course Dynamics: Trajectories and Transitions, Vol 1. Ithaca New York Cornell University Press; 1985:23-49.

[13] Thornberry TP. Three generation Studies: Methodological Challenges and Promise. Springer International Publishing; 2016:571-96.

[14] Thornberry TP, Knight KE, Lovegrove PJ. Does maltreatment beget maltreatment? A systematic review of the intergenerational literature. Trauma Violence Abuse 2012;13:135-52.

[15] Knight KE, Menard S, Simmons SB. Intergenerational continuity of substance use. Subst Use Misuse 2014;49:221-33.

[16] Kerr DCR, Tiberio SS, Capaldi DM. Contextual risks linking parents' adolescent marijuana use to offspring onset. Drug Alcohol Depend 2015; 154:222-8.

[17] Bailey JA, Hill KG, Guttmannova K, et al. Associations between parental and grandparental marijuana use and child substance use norms in a prospective, three-generation study. J Adolesc Heal 2016;59:262-8.

[18] Auty KM, Farrington DP, Coid JW. The intergenerational transmission of criminal offending: Exploring gender-specific mechanisms. Br J Criminol 2015:azv115.

[19] Craig L. Does father care mean fathers share? A comparison of how mothers and fathers in intact families spend time with children. Gend Soc 2006;20:259-81.

[20] Kim HK, Capaldi DM, Pears KC, et al. Intergenerational transmission of internalising and externalising behaviours across three generations: Gender-specific pathways. Crim Behav Ment Heal 2009;19:125-41.

[21] Serbin LA, Karp J. The intergenerational transfer of psychosocial risk: Mediators of vulnerability and resilience. Annu Rev Psychol 2004;55:333-63.

[22] Thornberry TP, Freeman-Gallant A, Lizotte AJ, et al. Linked lives: The intergenerational transmission of antisocial behavior. J Abnorm Child Psychol 2003;31:171-84.

[23] Henry KL, Thornberry TP, Lee RD. The protective effects of intimate partne relationships on depressive symptomatology among adult parents maltreated as children. J Adolesc Health 2015;57:150-6.

[24] Thornberry TP. Life course continuity and change in antisocial behavior final report for grant \#5R01MH063386 Prepared for the national Institute of mental health, Bethesda, MD. 2013.

[25] Odgers CL, Caspi A, Nagin DS, et al. Is it important to prevent early exposure to drugs and alcohol among adolescents? Psychol Sci 2008;19: $1037-44$.
[26] Substance Abuse and Mental Health Association (SAMSA). Age of substance use initiation among treatment admissions in aged 18 to 30 . Available at: http://www.samhsa.gov/data/sites/default/files/WebFiles_TEDS_SR142_ AgeatInit 07-10-14/TEDS-SR142-AgeatInit-2014.pdf. Published 2014 Accessed March 28, 2016.

[27] Robins LN, Cottler LB, Bucholz KK, et al. Diagnostic interview Schedule for the DSM-IV (DIS-IV). 2000.

[28] Muthen LK, Muthen BO. Mplus User's Guide. 6th edition. Los Angeles: Muthén \& Muthén; 2010.

[29] MacKinnon DP. Introduction to statistical mediation analysis. 2008.

[30] Rubin DB. Multiple Imputation for Nonresponse in Surveys. 1987.

[31] The White House. A drug policy for the 21st Century. Available at: https:// www.whitehouse.gov/ondcp/drugpolicyreform. Published 2014. Accessed March 28, 2016.

[32] Moffitt TE, Caspi A, Rutter M, Silva PH. Sex Differences in antisocial Behavior: Conduct disorder, delinquency, and Violence in the Dunedin longitudinal study. Cambridge: Cambridge University Press; 2001.

[33] Costello EJ, Erkanli A, Federman E, Angold A. Development of psychiatric comorbidity with substance abuse in adolescents: Effects of timing and sex. J Clin Child Psychol 1999;28:298-311.

[34] Capaldi DM, Tiberio SS, Kerr DCR, Pears KC. The relationships of parental alcohol versus tobacco and marijuana use with early adolescent onset of alcohol use. J Stud Alcohol Drugs 2016;77:95-103.

[35] Washburn IJ, Acock A, Vuchinich S, et al. Effects of a social-emotional and character development program on the trajectory of behaviors associated with social-emotional and character development: Findings from three randomized trials. Prev Sci 2011;12:314-23.

[36] Botvin GJ, Baker E, Dusenbury L, et al. Preventing adolescent drug abuse through a multimodal cognitive-behavioral approach: Results of a 3-year study. J Consult Clin Psychol 1990;58:437-46.

[37] Sun W, Skara S, Sun P, et al. Project towards no drug abuse: Long-term substance use outcomes evaluation. Prev Med 2006;42:188-92.

[38] Dishion TJ, Stormshak EA, Kavanagh KA. Everyday parenting: A Professional's Guide to Building family Management Skills. Champaign: Research Press; 2012.

[39] Spoth RL, Randall GK, Trudeau L, et al. Substance use outcomes 51/2 years past baseline for partnership-based, family-school preventive interventions. Drug Alcohol Depend 2008;96:57-68. 